\section{Unborn wasps fumigate their dinner}

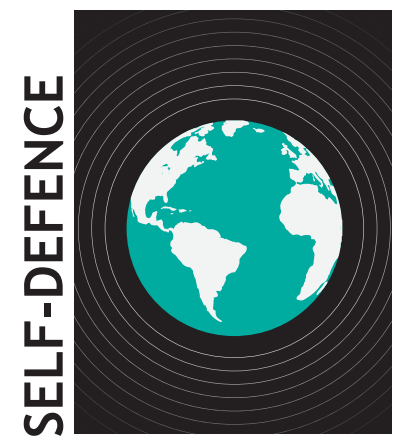

When the leftover food in my fridge turns into a microbiology experiment, I simply throw it out and get a fresh meal elsewhere. But not all animals have this luxury. Some only get a single meal and, if it rots, the animal starves and dies. Such is life for the larva of the European beewolf, whose parent provides it with a single paralysed bee to literally eat alive as it develops. The problem is that the larva and the bee are buried in an underground chamber surrounded by microbes that are as eager to eat the bee as the larva is. So how do beewolves keep their food clean? As elegantly shown by Erhard Strohm from the University of Regensburg in Germany and his colleagues from Mainz and Jena, they fumigate it.

If a paralysed bee is placed into a beewolf brood cell, it quickly turns into a fuzzy tennis ball covered with fungus. But this rarely happens if a beewolf properly does its job before abandoning its offspring. Strohm and his colleagues have shown over many years that beewolves use a series of antiseptic strategies to keep their larval resource fungus-free. First, they embalm the bee in a waxy resin that delays fungal growth. Second, they inoculate the brood cell with symbiotic bacteria that produce a potent antifungal drug. But these do not cover all eventualities, because the wax is only partially effective and the bacteria only protect older larvae in cocoons.

The hint that something more was at play came from the conspicuous odour of the brood cells themselves, notably cells that also contained a wasp egg. The team tested eggs and found that they secrete huge quantities of a pungent, and highly toxic, volatile gas called nitric oxide. Nitric oxide is used industrially to fumigate fruit against fungal contamination, so the team hypothesized that the eggs were using it in the same way to protect their bee prey. To test this, they placed bees with and without an egg into a brood cell and counted how many rotted. As predicted, the bees with individual eggs were protected from fungi. And, if they added a second bee - not in contact with the egg - this was decontaminated too, proving that protection was due to the volatile gas.

A nitric oxide fumigant is the perfect solution to the problem faced by these wasps. It fills all spaces in the brood cell where fungi hide and can dissolve into the water droplets on the surface of the bee, giving rise to nitrogen dioxide, yet another antimicrobial agent. It can also linger after a well-timed pulse of production, much like an unfortunate odour in a car with the windows up. Finally, its effects are general because nitric oxide can prevent the growth of a broad range of possible fungal contaminants.

But these advantages have to work in the context of the wasp's other defences and it seems they do. When the team combined waxy embalming with egg fumigation of their unfortunate bee victims, they found that the combination worked better than either alone. And importantly, although for unknown reasons, nitric oxide does not inhibit the growth of the symbiotic bacteria that make up the beewolf's third line of defence. The authors speculate that the bacteria may be protected in the brood cell or may have evolved resistance. Another possibility is that nitric oxide may be less active against bacterial spores, but this remains to be tested.

Parents go to great lengths to protect their offspring, but kids eventually have to fight their own battles. This amazing system shows that the unborn can also get in on the action of self-defence. Even eggs want a clean meal.

doi:10.1242/jeb.192997

Strohm, E., Herzner, G., Ruther, J., Kaltenpoth, M. and Engl, T. (2019). Nitric oxide radicals are emitted by wasp eggs to kill mold fungi. eLIFE 8 e43718. doi:10.7554/eLife.43718

Daniel E. Rozen (0000-0002-7772-0239)

Leiden University d.e.rozen@biology.leidenuniv.nl

\section{Singing seals imitate human speech}

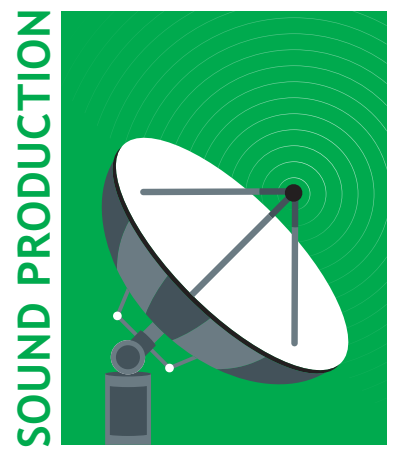

Have you ever wondered which animals learn to communicate with sounds and how? We can precisely control our vocal tract and vocal cords when learning new sounds. But the capacity for 'vocal learning' is extremely rare; fewer than 100 attested species with only a dozen mammals are capable of learning how to produce sounds for communication. For example, cats, dogs and chimpanzees cannot learn vocalizations. Amanda Stansbury and Vincent Janik from the University of St Andrews, UK, tested whether grey seals (Halichoerus grypus) can be trained to learn new sounds by imitation.

The researchers trained a grey seal, named Zola, to copy three sounds played through a loudspeaker. To make the task easier for Zola, these sounds were recordings of her own calls, which had been shifted in frequency to sound higher or lower. Every time Zola reproduced one of the sounds at the correct pitch, she received a fish reward. After training, Zola could copy sequences of up to 10 sounds varying in pitch, most of which she had not heard before. However, when the team analysed 
the audio recordings, they discovered that instead of modifying the vibration frequency of her vocal folds - as humans do when singing - Zola was altering the shape of her vocal tract, similar to the way we change the shape of our mouths when going from 'bath' to 'boot'. She could even imitate motifs such as the 'Star Wars' theme and 'Twinkle little star'; the videos are a must-watch. So, Zola the 'singing seal' could imitate tunes by performing vocal tract movements that we humans usually make when speaking.

Stansbury and Janik then trained two more seals, Janice and Gandalf, to imitate five vowel sounds - similar to those found in (British English) 'bath', 'may', 'meet', 'thought' and 'boot' - to assess the animals' ability to control their vocal tracts. To make the task more manageable, Stansbury and Janik produced the vowel sounds by modifying the seals' own calls and trained Janice and Gandalf to match these modified calls. Eventually, both seals could reliably imitate the five vowels by emphasizing either the lower or higher frequencies. The seals had changed their voices to sound deeper (as in 'boot') or more acute (as in 'meet'), so that they no longer sounded like other seals. They had learned how to produce new sounds instead of 'recycling' sounds they could already produce.

Why is this story scientifically interesting? Stansbury and Janik provide experimental evidence that it is possible for some mammals other than humans to learn to produce new sounds via modifications of their vocal tracts. This is something we humans do constantly when learning our mother tongue or a new language. Currently, many researchers study bird song production to help us understand human speech, but now a much closer relative - seals, with their human-like vocal tracts and learning abilities - could be a perfect alternative model species for learning about human speech and song thanks to our similar anatomy. In addition, it is possible to measure the brain activity of seals, potentially allowing us to identify which areas of the brain are key for seal vocal imitation.

In brief, seals may have much to teach us about human speech and song. Seal scientists are beginning to listen and you are all welcome to join in too.

doi:10.1242/jeb.208447
Stansbury, A. L. and Janik, V. M. (2019). Formant modification through vocal production learning in gray seals. Curr. Biol. doi:10.1016/j.cub.2019. 05.071

Andrea Ravignani (0000-0002-1058-0024) Vrije Universiteit Brussel \& Sealcentre Pieterburen andrea.ravignani@gmail.com

\section{Sun and wind cues guide dung-rolling beetles across the savannah}

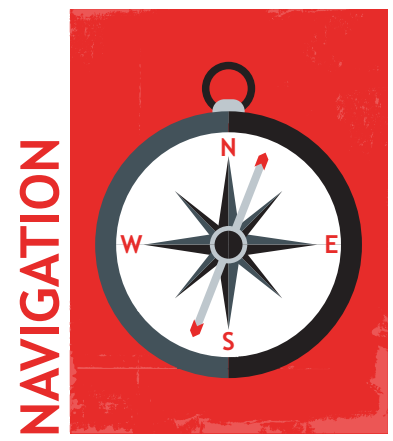

South African dung beetles compete fiercely for the chance to eat the poop of other animals. When a beetle comes across a pile of dung in the savannah, it quickly cuts off a piece, rolls it into a ball, and heads off in reverse with its stinky prize, away from other hungry beetles. To efficiently escape with their trophy, the beetles move in a straight line, orienting themselves with celestial objects such as the sun or moon. However, new research from Marie Dacke and colleagues from Swedish, German and South African universities suggest that the beetles can also steer based on changes in wind direction.

First, Dacke and colleagues tested whether beetles in their natural habitat steer dung balls in a straight line using cues from the wind in addition to the celestial cues they are known to use. The researchers tested the beetles when the sun was at three different positions above the horizon - low (15-30 deg), medium (45-60 deg) and high (>75 deg) - and observed whether they continued to roll their dung balls in the same direction in response to a $180 \mathrm{deg}$ artificial change in wind direction. When the sun was at its highest elevation and the experimenters switched the direction of the wind, the beetles started rolling their dung balls in the opposite direction. However, when the sun was at the lower elevations, the beetles kept rolling their balls in the same direction no matter how the wind changed. This suggests that the beetles orient themselves based on a single cue at a time, either the sun or the wind, and that they might, in fact, have a 'wind compass' they use when the sun is high in the sky.

The team then took the beetles back to the lab to better understand how they coordinate using wind and sun cues. First, the scientists changed the number of sensory inputs the beetles received between each roll of their dung balls forward: they provided the beetles with multiple inputs on their first rolls (wind from a fan and a bright light to mimic the sun, set perpendicular to each other). They then removed one of the inputs during the second revolution of the dung ball - leaving the insects to navigate either by artificial 'wind' or a fake 'sun' alone. In response, the beetles continued rolling their dung balls in straight lines despite switching from multiple to individual sensory inputs, which was surprising, as under natural conditions in the savannah the beetles seemed to position themselves based on one sensory input at a time. However, the beetles' ability to continue moving in a straight line suggests that they store directional information from both the wind and the sun in their internal compass systems. The researchers then tested whether orientation information from the sun and wind sensory systems can be stored and exchanged between different brain areas. They realised that the beetles were able to depend on directional information from the sun and moon collected at different times, suggesting that information from the two systems is shared somewhere in the brain.

Dacke and colleagues are the first to show that wind is important for helping South African dung beetles roll away from hungry competitors with their dinner. Additionally, their research highlights that information from the wind and the sun might be stored in the same place in the beetles' brains. For an animal that feasts on other animals' poop, the South African dung beetle has quite a sophisticated navigation system.

doi:10.1242/jeb.193003

Dacke, M., Bell, A. T. A., Foster, J. J., Baird, E. J., Strube-Bloss, M. F., Byrne, M. J. and el Jundi, B. (2019). Multimodal cue integration in the dung beetle compass. Proc. Natl. Acad. Sci. USA 116, 14248-14253. doi:10.1073/pnas. 190430 8116 
Gina Mantica (0000-0001-5329-3896)

Tufts University

gina.mantica@tufts.edu

\section{Pain insensitivity: subtle changes make a huge difference}

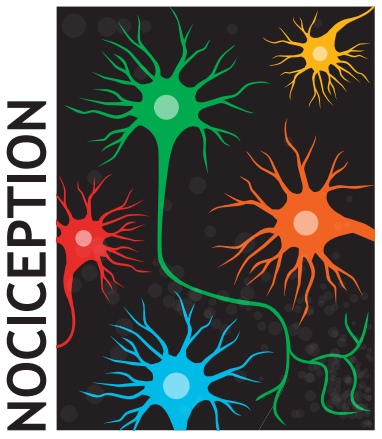

Some years ago, after putting out the recycling, I had the unpleasant discovery that my dog had got into my bag of jalapeño potato chips and thrown up all over the floor. They obviously hadn't sat well with him. As I cleaned up the mess, I pondered why my dog was so quick to expel the snacks, whereas I could happily eat a whole bag in a single sitting. Evidently, the same question occurred to Ole Eigenbrod of the Max Delbrück Center, Germany, who teamed up with a group of international collaborators to explain why some animals tolerate certain substances, whereas others do not.

The nervous system perceives pain via specialized neurons (nociceptors) that respond to substances ranging from lethal venom to benign pepper. However, even closely related species do not respond with equal intensity to the same compounds, thanks to their different evolutionary trajectories. For this reason, Eigenbrod and colleagues studied pain insensitivity in nine species of related African burrowing rodents, which are separated by 7-55 million years of evolutionary history, by evaluating their reactions to three noxious molecules: capsaicin (which makes jalapeños spicy), hydrochloric acid (found in acidic fruits) and AITC (a pungent molecule in many root vegetables). After dabbing a stinging molecule onto each animal's footpad, the authors assessed how much pain they experienced by measuring the time each rodent spent licking its paw. They also investigated the molecular components of the rodent's pain neurons, to identify the specific differences that underlie pain insensitivity.

The researchers initially found that the pain neurons of all nine rodent species possess the receptor molecules that should allow them to detect the three noxious molecules. However, some of the species did not exhibit pain in response to these compounds. For instance, highveld molerats did not feel AITC dabbed onto their paws, even though there are neuron receptors that should bind the noxious chemical and potentially trigger pain. The team attributes this insensitivity to the overexpression of a gene for a sodium leak ion channel that short circuits the pain neurons and prevents them from transmitting signals. The team suspects that AITC resistance stems from the highveld mole-rats sharing their burrows with aggressive Natal droptail ants, whose excruciatingly painful stings activate the AITC receptor. Clearly, it was advantageous for the rodents to evolve immunity to the stings.

The team also observed that East African root-rats, Cape mole-rats and naked mole-rats were completely oblivious to the hydrochloric acid dabbed onto their paws. The authors found that the pain neurons of these three species possess a sodium ion channel with the same two amino acid alterations. These changes prevent hydrochloric acid from activating the ion channel, rendering the neurons insensitive to the acid and unable to trigger pain. Considering that the three species are separated by 40 million years of evolutionary history, these modifications are a classic case of convergent evolution, allowing all three species to tolerate hydrochloric acid.

Finally, the researchers noted that Natal and naked mole-rats are impervious to the spicy jalapeño capsaicin and believe the rodents' evolved insensitivity is due to them inhabiting deep, oxygen-poor burrows, where $\mathrm{CO}_{2}$ levels can reach very high levels. Given that inhaling large amounts of $\mathrm{CO}_{2}$ can cause a great deal of pain, it is likely that the mole-rats adapted to their underground lifestyle by blunting most of their pain responses.

Although the nine rodent species are closely related, they each live in very different geographical environments and have faced different selection pressures that have led to their varying degrees of pain sensitivity. It appears that evolution has several lessons to teach us about the various molecular mechanisms that can mediate pain

doi:10.1242/jeb.192989

Eigenbrod, O., Debus, K. Y., Reznick, J., Bennett, N. C., Sánchez-Carranza, O., Omerbašić, D. Hart, D. W., Barker, A. J., Zhong, W. Lutermann, H., Katandukila, J. V., Mgode, G. Park, T. J. and Lewin, G. R. (2019). Rapid molecular evolution of pain insensitivity in multiple African rodents. Science 364, 852-859. doi:10. 1126/science.aau0236

Ilan Ruhr (0000-0001-9243-7055)

The University of Manchester ilanruhr@manchester.ac.uk

\section{Andean birds with slow metabolism live longer}

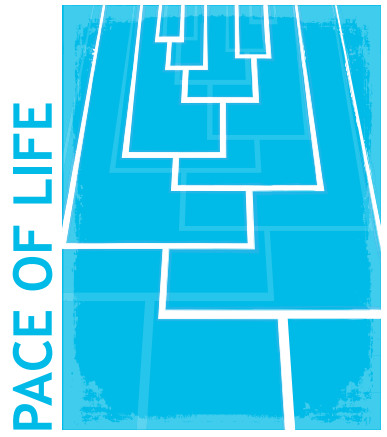

Metabolism - the chemical process that generates energy for cells to function - is so fundamental to existence that it has been dubbed 'the fire of life'. However, the debate over how the rate of metabolism can influence an animal's survival has burned on for decades. The 'pace of life' theory predicts that species with high metabolic rates accrue more damage and succumb to death earlier than animals with slower metabolism. Whilst this makes sense in theory - a gently burning flame lasts longer than a firework - experimentally proving it has been a tricky business.

To shed light on how metabolic rate correlates with survival in wild tropical birds, a team led by Micah Scholer from the University of British Columbia, Canada, studied a diverse cohort of 37 species in the Peruvian Andes, from the humid foothills up to $3000 \mathrm{~m}$ altitude. The group caught birds in mist-nets and released them, tagged but unharmed, at different sites over several years to assess annual survival. They then combined this dataset with previously acquired measurements of basal metabolic rate of the same species from the same field sites - achieved by measuring oxygen 
consumption in resting animals - in a sophisticated mathematical model to dissect the interplay between metabolism, survival and habitat.

Survival varied greatly between different species; in some cases, annual survival exceeded $70 \%$, whereas in the most vulnerable species, less than half this proportion survived each year. The team further revealed that, although metabolic rate was similar between birds inhabiting different altitudes, montane species exhibited lower survival than those from the foothills. Avian life at high altitude is something of a double-edged sword; the harsher, drier environment provides challenges, yet this also discourages predators. It appears here that the costs of living at altitude outweighed the benefits of lower predation, although the research team was unable to exclude the possibility that the high-altitude birds were emigrating from the study site instead of perishing.

The standout result was that, irrespective of altitude, the species with the lowest metabolic rate - or the slowest pace of life - indeed exhibited the highest rate of survival. Whether annual survival per se equates to evolutionary success is doubtful, because even the species with the lowest survival rate are, by definition, successful enough to prosper today. Apparently, they are able to reproduce effectively in their short but busy lifetime, which may be fuelled by their high metabolic rate. The fact that species with both short and long lifespans persist suggests that neither strategy is optimal.

By revealing a well-defined link between metabolism and survival, this study not only represents a great advance for environmental physiology but also helps pave a path for future work. For example, researchers could measure metabolic rates in animals' natural environments, which is tricky but technically feasible, to allow us to describe how metabolism changes seasonally in the different habitats. Ultimately, this will help paint an ever-clearer picture of how and why survival and metabolism vary between different species.

doi:10.1242/jeb.193011

Scholer, M. N., Arcese, P., Puterman, M. L. Londoño, G. A. and Jankowski, J. E. (2019). Survival is negatively related to basal metabolic rate in tropical Andean birds. Functional Ecology 259, R333. doi:10.1111/1365-2435. 13375

William Joyce (0000-0002-3782-1641)

University of Ottawa william.joyce@bios.au.dk 